\title{
Impacto de gastroenteritis severa por rotavirus en niños chilenos menores de 3 años de edad
}

\author{
Rodrigo Vergara $\mathrm{F}^{1,2}$, María S Navarrete ${ }^{3}$, Ernesto $\mathrm{N}_{\text {úñez }}{ }^{4}$, \\ Lorena Escobara, Sofía Navarro Ga, G uillermo Venegas $\mathrm{E}^{4}$, \\ Patricia Schuffeneger ${ }^{5}$, Miguel 0 'Ryan ${ }^{6}$. \\ Incidence of severe rotavirus \\ gastroenteritis among Chilean children \\ under three years of age
}

Background: Rotavirus infections account every year in Chile, for approximately 53,000 emergency consultations and 8,000 hospital admissions among children under three years of age. Aim: To estimate incidence rates of severe rotavirus gastroenteritis in children $<3$ years of age, living in the V and VIII Regions and to identify the predominant viral serotypes. Material and methods: A prospective hospital-based surveillance for severe gastroenteritis was implemented in public and private hospitals of Viña del Mar and Valparaíso (Region V) and of Chiguayante, Concepción, Penco, San Pedro de la Paz, Talcahuano and Tomé (Region VIII). All children $<3$ years of age residing in the districts, who consulted for severe gastroenteritis requiring oral or intravenous rehydration (equivalent to WHO plan B or C), or who were admitted to the hospital, were enrolled. Demographic and clinical information and a stool sample were obtained. Rotavirus was detected by ELISA and positive samples were serotyped by ELISA or real time PCR. Results: Between January 23 and June 30, 2003, a total of 760 children were recruited. Among these, 343 (45\%) were admitted to the hospital. Stool samples were collected from 433 children. Among these, 214 were positive for rotavirus (49.4\%). Overall, monthly disease incidence rates were 124/100,000 in V Region, and 114/100,000 in VIII Region. The predominant serotype was G4. Conclusions: Rotavirus was responsible for nearly half of the severe gastroenteritis episodes among children $<3$ years, during a predominantly G4 serotype season. Every year, approximately one every 70 children $<3$ years will have a severe rotavirus gastroenteritis episode (Rev Méd Chile 2007; 135: 975-81).

(Key words: Child; Gastroenteritis; Rotavirus)

Recibido el 14 de diciembre, 2005. Aceptado el 10 de julio, 2006.

Fuente de apoyo financiero: Este estudio contó con el apoyo de GlaxoSmithKline (GSK), que financió los honorarios de todo el personal responsable de la conducción del estudio y los gastos de laboratorio. Miguel O'Ryan recibe honorarios como consultor para el desarrollo de la vacuna rotavirus humana atenuada de GSK. Personal de GSK participó activamente en el diseño del estudio. No hubo participación de GSK en la recolección de los datos. En el análisis e interpretación de los datos, como en la preparación, revisión y aprobación del artículo, participaron todos los autores, incluyendo María Soledad Navarrete, gerente médico de GSK Chile. No hubo restricción de GSK a elección de revista.

${ }^{1}$ Escuela de Medicina, Universidad de Valparaíso. ${ }^{2}$ Hospital Carlos van Buren, Valparaíso. ${ }^{3}$ Glaxo SmithKline Chile. ${ }^{4}$ Escuela de Medicina, Universidad de Concepción. ${ }^{5}$ Hospital Gustavo Fricke, Viña del Mar. ${ }^{6}$ Instituto de Ciencias Biomédicas, Facultad de Medicina de la Universidad de Chile. Santiago de Chile.

aEnfermera.

Correspondencia a: Rodrigo Vergara Fisher. Escuela de Medicina, Universidad de Valparaíso. Hontaneda 2653 Valparaíso,

Chile. Fax 56-32-507375. E mail: rodrigo.vergara@uv.cl 
$\mathrm{R}$ otavirus fue descubierto en 1973 y fue rápidamente reconocido como el principal agente causal de gastroenteritis severa en niños ${ }^{1}$. Se trata de un virus ARN, con genoma fragmentado, para el cual se han descrito diversos serotipos, predominando el serotipo G1 en la mayoría de las regiones del mundo. Estudios de cohortes han permitido observar que prácticamente todos los niños presentan al menos una infección por rotavirus antes de cumplir los 5 años, y sobre $90 \%$ antes de los 3 años, independientemente del nivel socioeconómico ${ }^{2,3}$. Cada año, rotavirus causa alrededor de 111 millones de episodios de gastroenteritis (GE) aguda que requieren cuidado domiciliario, 25 millones de consultas médicas, 2 millones de hospitalizaciones y, al menos, 440.000 muertes en niños menores de 5 años. El 82\% de estas muertes ocurren en los países más pobres del mundo, primordialmente en África y Asia ${ }^{4}$.

En Chile, un estudio estimó que anualmente rotavirus sería responsable de alrededor de 53.000 consultas médicas de urgencia y 8.000 hospitalizaciones en niños menores de 3 años ${ }^{5}$. Estas estimaciones se basaron en datos prospectivos obtenidos a partir de las consultas en sólo un hospital de la Región Metropolitana. Si bien no se ha estudiado dirigidamente, en Chile es infrecuente que ocurran muertes debido a diarrea por cualquier causa ${ }^{5}$.

Recientemente se ha registrado en Chile una vacuna contra el rotavirus en base a un virus atenuado, que ha demostrado ser eficaz y segura. La eficacia protectora de esta vacuna contra las formas más severas de infección, incluyendo hospitalización, supera el 85\% y contra gastroenteritis de cualquier gravedad es de hasta 70\%6-8. La definición de la incorporación eventual de una nueva vacuna a los Programas de Vacunación en Chile requerirá del conocimiento lo más acabado posible del impacto de enfermedad asociada a rotavinus, especialmente como causa de diarreas severas que requieren atención médica de urgencia u hospitalización, y la evaluación de su costo efectividad ${ }^{9}$ (Constenla D. Sometido a publicación).

El presente estudio tuvo como objetivo determinar el impacto de la GE severa en niños menores de 3 años en dos regiones de Chile ( $\mathrm{V}$ y VIII). Un objetivo secundario fue la identificación de los serotipos de rotavirus que causaron infección durante el período de estudio.

\section{MATERIAL Y MÉTODO}

Descripción de las áreas de estudio. La VIII región es la segunda más poblada del país, con 1.861 .562 habitantes distribuidos en 52 comunas. Para los fines de este estudio, se implementó un sistema de vigilancia que abarcó a la población perteneciente a las comunas de Chiguayante, Concepción, Penco, San Pedro de la Paz, Talcahuano y Tomé, las que de acuerdo al censo del año 2002 alojan 726.614 habitantes (39\%), de los cuales 52.412 son menores de 5 años. La $\mathrm{V}$ región es la tercera más habitada del país, con 1.539.852 habitantes distribuidos en 38 comunas. En esta región, el sistema de vigilancia abarcó a las dos principales comunas de la región, Viña del Mar y Valparaíso, las que alojan 562.913 residentes (37\% de la región), de los cuales 36.793 corresponden a niños menores de 5 años de edad ${ }^{10}$.

$\mathrm{Al}$ igual que el resto del país, la mayoría de la población (72\%) de estas dos regiones, recibe atención de salud a través del sistema estatal (FONASA), mientras que $28 \%$ lo hace a través del sistema de seguros privados o Instituciones de Salud Previsional (ISAPRES) ${ }^{11}$. La población pediátrica de las comunas bajo vigilancia de la VIII región, se atiende en los hospitales públicos Regional Guillermo Grant Benavente de Concepción, Higueras de Talcahuano, Hospital Tomé y el Hospital Lirquén y en 2 clínicas privadas, el Sanatorio Alemán y la Clínica Francesa. En la V región, la población infantil se atiende principalmente en 2 hospitales públicos de atención terciaria, los hospitales Gustavo Fricke en Viña del Mar y Carlos van Buren en Valparaíso, y en un centro de atención privada, el Hospital de Niños y Cunas de Viña del Mar. Todos estos centros se mantuvieron bajo vigilancia durante el período de estudio.

Diseño de la vigilancia. Los centros descritos fueron vigilados activamente durante el período comprendido entre el 23 de enero y el 30 de junio de 2003, permitiendo captar a todo niño menor de 3 años de edad que consultó por gastroenteritis severa. En cada centro se contaba con personal del estudio los 7 días de la semana y las 24 horas del día.

Se definió como gastroenteritis severa (GES) a todo caso de diarrea (tres o más deposiciones no formadas en un período de $24 \mathrm{~h}$ ), con o sin 
vómitos, que cumpliera una de las 2 condiciones siguientes: a) que al examen clínico presentara deshidratación según el médico tratante y al cual se le indicara hidratación oral o endovenosa en el centro asistencial (equivalentes a plan $\mathrm{B}$ o $\mathrm{C}$ de la Organización Mundial de la Salud (OMS) o b) que se hospitalizara por causa de una gastroenteritis. Se incluyó como caso a todo niño que cumpliera con esta definición y que además 1) tuviera su domicilio en el área de estudio, 2) tuviera menos de 3 años de edad al momento de la consulta y 3) cuyos padres dieran su consentimiento informado para la participación del niño en el estudio.

El personal encargado de la vigilancia tenía la responsabilidad de detectar, en las salas de atención, de observación y de hospitalización a todo niño que cumpliera con los requisitos para ingresar al estudio, solicitar el consentimiento informado a los padres o tutores legales, y recolectar información relevante mediante entrevista a los acompañantes y revisión de fichas clínicas y hojas de atención médica y de enfermería.

A los acompañantes de los niños ingresados se les realizó una breve entrevista con formato predefinido, que incluyó datos demográficos (edad, sexo, comuna de residencia) e información clínica acerca de la presentación y evolución de la enfermedad previo al enrolamiento (presencia y duración de fiebre, vómitos y diarrea, medicamentos prescritos previo a la consulta, tipo de alimentación recibida). Se recolectó una muestra de deposiciones en los pacientes que presentaron emisión espontánea durante el período que permanecieron en el centro asistencial. Los niños fueron observados por personal del estudio hasta su alta del centro asistencial.

Manejo de muestras. Las muestras de deposiciones fueron almacenadas en refrigerador a temperaturas entre $2^{\circ}$ y $8^{\circ} \mathrm{C}$, por un máximo de 3 días, después de lo cual fueron transportadas a un laboratorio local donde se prepararon alícuotas para almacenamiento a $-20^{\circ} \mathrm{C}$. Estas fueron enviadas posteriormente al Laboratorio de Microbiología de la Escuela de Medicina de la Universidad de Chile para identificación de rotavirus mediante ELISA, utilizando anticuerpos contra la proteína VP6 de rotavirus. Las muestras positivas fueron serotipificadas mediante ELISA o PCR según metodología previamente descrita ${ }^{12,20}$.
Registro y análisis de datos. La información clínica, de laboratorio y de las encuestas fueron introducidos en una base de datos local y en una base de datos de ingreso remoto (RDE, GlaxoSmithKline Biologicals, Bruselas, Bélgica). Para el análisis descriptivo y los tests estadísticos realizados se utilizó el software Stata 5.0. (Stata Corporation, Texas USA). Para los tests con variables cualitativas se aplicó la prueba de chi cuadrado y para variables cuantitativas la prueba de ANOVA. Se calcularon las tasas mensuales de GE y de GE por rotavirus para las dos áreas evaluadas. Para la estimación del denominador se utilizó la población total de nacidos vivos al año 2002 en las comunas comprendidas en el área de estudio ${ }^{13}$.

Aspectos éticos. El estudio fue aprobado por un Comité ad-hoc convocado por la Unidad de Bioética del Ministerio de Salud, cuya estructura y funcionamiento están en conformidad con la declaración de Helsinki y con las pautas de Buenas Prácticas Clínicas de la $\mathrm{ICH}^{14,15}$.

\section{RESULTADOS}

Los equipos de vigilancia identificaron 1.133 casos de gastroenteritis severa, potencialmente elegibles, durante los 158 días que duró el estudio. De éstos, 24 sujetos no ingresaron porque rehusaron participar y otros 349 porque no cumplían con uno o más criterios de selección (fuera del rango de edad, lugar de residencia en comunas ajenas al estudio, ausencia de deshidratación, $<3$ deposiciones en $24 \mathrm{~h}$ ).

En consecuencia, ingresaron al estudio 760 participantes elegibles. Se obtuvo una muestra de deposiciones por emisión espontánea en 433 (57\%) de los sujetos incluidos, cuyas características demográficas y clínicas están resumidas en la Tabla 1. Se observó una ligera predominancia del sexo masculino, independientemente del agente causal. En 214 sujetos (49\%) se identificó el rotavirus como el agente causal de la GES ( $43 \%$ en la V Región y 58\% en la VIII Región). La edad promedio de los niños con GES por rotavirus fue de 16 meses, con un rango que varió entre 1 y 35 meses. Los episodios positivos para rotavirus se caracterizaron por una significativa mayor presencia de vómito, necesidad de hidratación endove- 
Tabla 1. Características de los participantes según presencia de rotavirus en deposiciones

\begin{tabular}{|lrrrr|}
\hline & Rotavirus + & Rotavirus - & Test no realizado & \\
& $\mathrm{N}(\%)$ & $\mathrm{N}(\%)$ & $\mathrm{N}(\%)$ & $\mathrm{P}$ \\
\hline $\mathrm{N}$ & 214 & 219 & 327 & \\
Sexo Fem/Masc (\%) & $(42 / 58)$ & $(40 / 60)$ & $(45 / 55)$ & NS* $^{*}$ \\
Edad (meses) \pm DS & $16,1 \pm 7,4$ & $16,7 \pm 9,8$ & $17,8 \pm 8,9$ & NS* \\
Presencia de vómito & $204(95)$ & $173(79)$ & $292(89)$ & $<0,0001^{*}$ \\
Diarrea con sangre & $5(2)$ & $26(12)$ & $7(2)$ & $<0,0001^{*}$ \\
Hospitalización & $124(58)$ & $130(59)$ & $89(27)$ & NS* \\
Hidratación endovenosa & $191(89)$ & $167(76)$ & $235(72)$ & $<0,0001^{*}$ \\
Uso de antibióticos & $10(5)$ & $61(28)$ & $24(7)$ & $<0,0001^{*}$ \\
\hline
\end{tabular}

*comparando rotavirus (+) vs rotavirus (-)

nosa, una menor frecuencia de diarrea con sangre y menor uso de antimicrobianos en comparación con los episodios en que no se detectó rotavirus. Se hospitalizaron 343 niños (45\%), la indicación de hospitalización fue igual entre niños con y sin rotavirus en deposiciones. El grupo de niños en el que no se pudo realizar el test de detección de rotavirus, porque no presentaron emisión espontánea de deposiciones durante la estadía en el centro asistencial, presentó un perfil clínico intermedio, destacando una significativa menor fre- cuencia de hospitalización en comparación con niños rotavirus positivos y negativos ( $p<0,001$ ).

La Figura 1 muestra la distribución acumulada de casos en función de la edad del paciente al momento del diagnóstico. El 7,5\%,34\%, 67\% y $87 \%$ de los casos de GES por rotavirus ocurrieron antes de los 6, 12, 18 y 24 meses de edad, respectivamente. La Figura 2 muestra el número de episodios totales de GES y los casos positivos para rotavirus por mes. Hubo una disminución de casos totales de GES en los meses más fríos,

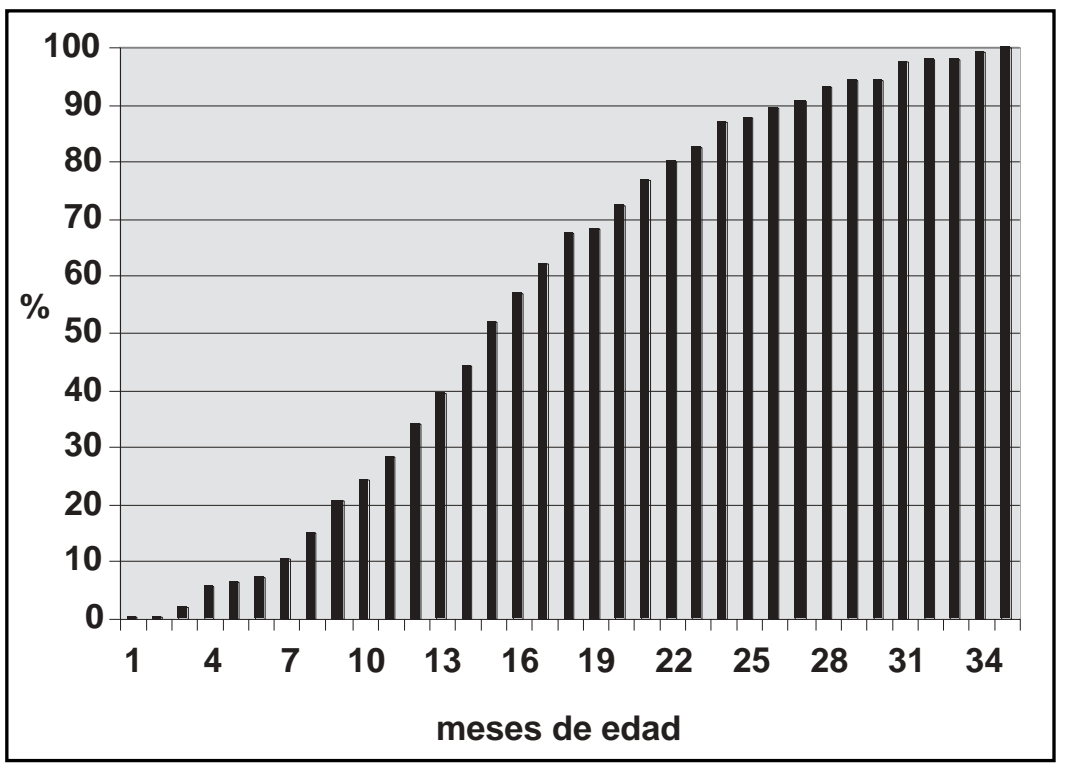

Figura 1. Distribución acumulada por edad de las gastroenteritis severas causadas por rotavirus, durante los meses de febrero a junio de 2003 en la V y VIII región, Chile. 


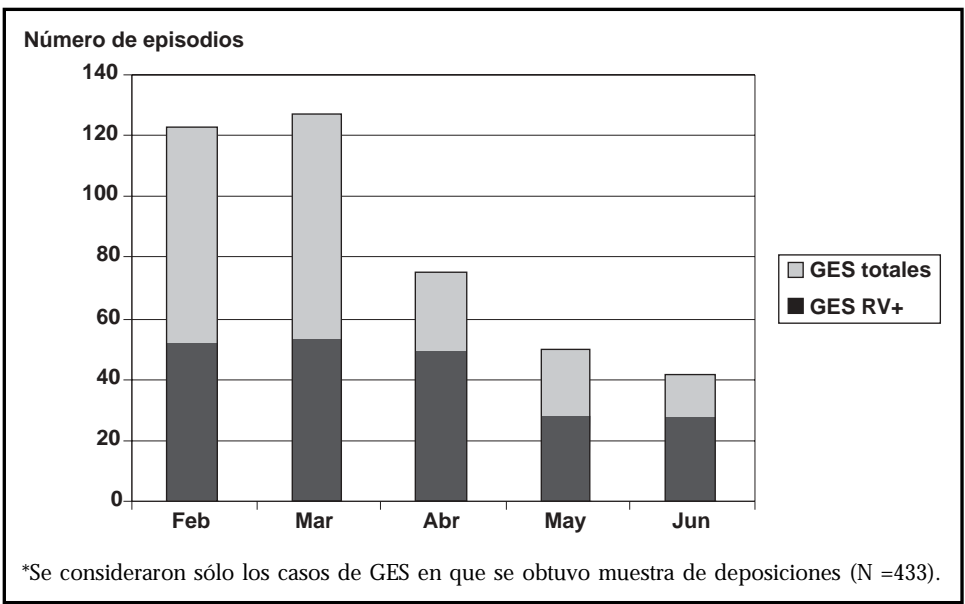

Figura 2. Episodios de gastroenteritis severas totales y causadas por rotavirus durante los meses de febrero a junio de 2003 en la V y VIII región, Chile.

principalmente a expensas de los casos rotavirus negativo. La proporción de los casos rotavirus positivos en relación a los episodios totales aumentó de 33\% a 58\% de febrero a junio.

Se logró tipificar 224 serotipos de rotavirus en las 214 muestras de deposiciones rotavirus positivas. Durante el período de 5 meses circularon rotavirus de serotipos G1, G2 y G4. El serotipo G4 fue el que se aisló con mayor frecuencia, encontrándose en 159 de los 224 rotavirus aislados en deposiciones (71\%). La distribución de los serotipos fue significativamente diferente en las dos regiones estudiadas (Tabla 2). El serotipo G4 presentó una frecuencia de $62 \%$ en la $\mathrm{V}$ región y de 82\% en la VIII región. El serotipo G1 presentó una frecuencia global de $17 \%$ distribuido en $24 \%$ en la
V región y 9\% en la VIII región. Sólo se identificó el serotipo G2 en un caso de Valparaíso, el que fue aislado en una muestra junto con rotavirus G4.

La tasa mensual promedio estimada de GES por rotavirus fue de 124 por 100.000 menores de 3 años para la $\mathrm{V}$ región y de 114 por 100.000 menores de 3 años para la VIII región (Tabla 3). Las tasas mensuales variaron entre 26 y 220 por 100.000 durante el período, destacando febrero y junio con las tasas más altas y bajas, respectivamente. La tasa mensual promedio estimada de hospitalización por GES por rotavirus fue de 40 por 100.000 menores de 3 años para la $V$ región y 59 por 100.000 para la VIII. Las tasas mensuales fluctuaron entre 16 y 109 por 100.000 durante el período, siguiendo un patrón similar a los casos de GES.

Tabla 2. D istribución de serotipos de rotavirus por región

\begin{tabular}{|c|c|c|c|c|c|c|c|c|c|c|}
\hline & \multicolumn{2}{|r|}{ G1 } & \multicolumn{2}{|c|}{$\mathrm{G} 2$} & \multicolumn{2}{|r|}{ G4 } & \multicolumn{2}{|c|}{$\begin{array}{c}\text { No } \\
\text { tipificables }\end{array}$} & \multirow{2}{*}{$\mathrm{P}$} & \multirow{2}{*}{$\begin{array}{c}\text { Total } \\
\text { pacientes/ } \\
\text { serotipos } \\
\text { aislados }\end{array}$} \\
\hline & $\mathrm{N}$ & (\%) & $\mathrm{N}$ & $(\%)$ & $\mathrm{N}$ & $(\%)$ & $\mathrm{N}$ & $(\%)$ & & \\
\hline V región & 28 & (24) & 1 & (1) & 72 & $(62)$ & 16 & (13) & 0,008 & 112/117 \\
\hline VIII región & 10 & (9) & 0 & (0) & 87 & (82) & 10 & (9) & & 102/107 \\
\hline Total & 38 & (17) & 1 & (0) & 159 & (71) & 26 & (12) & & $214 / 224^{*}$ \\
\hline
\end{tabular}

*En algunos especímenes se detectaron 2 serotipos. 


\section{Tabla 3. Estimación de impacto de gastroenteritis severa por rotavirus en la V y VIII regiones, C hile (febrero a junio 2003)}

\begin{tabular}{|c|c|c|c|c|c|}
\hline & $\begin{array}{c}\text { Consultas por } \\
\text { Gastroenteritis severa }\end{array}$ & $\begin{array}{c}\text { Con muestra } \\
\mathrm{N}(\%)\end{array}$ & $\begin{array}{c}\text { Positivas RV } \\
\text { N (\%) }\end{array}$ & $\begin{array}{l}\text { GES atribuibles a } \\
\text { rotavirus* }\end{array}$ & $\begin{array}{c}\text { Tasa mensual } \\
\text { promedio } \\
\text { estimada de GES } \\
\text { por RV** }\end{array}$ \\
\hline V región & 392 & $244(62)$ & $106(43)$ & 170 & 124 \\
\hline VIII región & 341 & $173(51)$ & $101(58)$ & 199 & 114 \\
\hline Total & 733 & 417 (57) & $207(50)$ & 369 & 119 \\
\hline
\end{tabular}

* Corresponde a la aplicación de la frecuencia obtenida en las GES con muestra a las consultas totales por GES.

* Tasas por 100.000 habitantes menores de 3 años.

\section{DisCUSIÓN}

Rotavirus se asoció a la mitad de los casos de gastroenteritis severa en niños menores de 36 meses de la V y VIII regiones durante los 5 meses de vigilancia prospectiva. Estos resultados son comparables a otros estudios nacionales e internacionales que detectan rotavirus en 30\%-70\% de los casos de gastroenteritis severa, acercándose a $50 \%$ cuando se requiere de hospitalización ${ }^{16,17}$. Los hallazgos clínicos asociados a rotavirus fueron los esperados de acuerdo con reportes previos, destacando la presencia de vómitos, ausencia de sangre en deposiciones y de una mayor necesidad de hidratación endovenosa ${ }^{18}$.

Una de las limitaciones de nuestro estudio es que sólo comprende 5 meses del año. Aun cuando lo ideal habría sido cubrir los 12 meses, creemos que es posible extrapolar los resultados a cifras anuales. Un estudio previo realizado durante 2 años en un hospital público de Santiago, demostró una estacionalidad poco marcada y con sólo un mes de ausencia de detección de rotavirus. Por otro lado, el período comprendido en nuestro estudio incluyó meses de alta y baja frecuencia de gastroenteritis. La tasa anual extrapolada de GES por rotavirus es de 1.428 por 100.000 menores de 3 años para las 2 áreas estudiadas, siendo semejantes en las 2 regiones (1.480 en la V región y 1.360 en la VIII región). La tasa anual estimada de hospitalizaciones por gastroenteritis por rotavirus fue de 648 por 100.000 menores de 3 años. Dicho de otra manera, cada año, 1 de cada 70 niños menores de 3 años presentaría una gastroenteritis severa por rotavirus y 1 de cada 154 requerirían hospitalización. Estos resultados son comparables a los obtenidos por O'Ryan y cols ${ }^{5}$, quienes estimaron que 1 de cada 136 requerían hospitalización. Se debe destacar, además, que la vigilancia fue realizada en los principales centros de atención (incluyendo privados y públicos) con cobertura de 24 h, 7 días a la semana por personal propio del estudio, lo cual dificulta la pérdida de sujetos. Como una medida de cautela para evitar aumentar espúreamente las tasas, se decidió utilizar el total de nacidos vivos para el año 2002 como denominador para el cálculo de tasas (esta cifra incluye a nacidos en el sistema público y privado y es mayor a la población estimada según el censo del año 2002). Usando este denominador, se tiende a subestimar y no sobreestimar la tasa de gastroenteritis severa por rotavirus.

Resultó sorprendente la mayor proporción de serotipo G4 en las dos regiones estudiadas. Este serotipo se había encontrado en forma esporádica en estudios realizados en Chile durante la última década $^{19,20}$, estando prácticamente ausente en regiones diferentes de la VIII. Este hallazgo no es, sin embargo, inesperado, ya que la circulación de los diferentes serotipos en el mundo no tiene un patrón definido, por lo que cabe esperar la alternancia en predominancia de serotipos en una región a lo largo de los años ${ }^{20}$. La predominancia de G4 en el año 2003 probablemente se debió a una reducida inmunidad poblacional anti-G4 secundaria a su baja circulación durante los años previos $^{18}$. 
Nuestro estudio reafirma la importancia de rotavirus como causa de gastroenteritis en nuestro país y lo identifica como la principal causa de gastroenteritis severa. Aporta, a nuestro parecer,

\section{REFERENCIAS}

1. Bishop RF, Davidson GP, Holmes IH, Ruck BJ. Viral particles in epithelial cells of duodenal mucosa from children with acute non-bacterial gastroenteritis. Lancet 1973; 2: 1281-3.

2. KapiKian AZ, Chanock RM. Rotaviruses. En B. N. Fields, et al. (ed.), Virology, $3^{\text {rd }}$ ed. LippincottRaven Press, New York 1996; 1657-1708.

3. Wilhelmi I, Román E, Sánchez-Fauquier A. Viruses causing gastroenteritis. Clin Microbiol Infect 2003; 9: 247-62.

4. Parashar UD, Hummelman EG, Breese JS, Miler MA, Giass RI. Global Illness and deaths caused by rotavirus disease in children. Emerg Infect Dis 2003; 9: 565-72.

5. O'Ryan M, Pérez-Schael I, Mamani N, Peña A, Salinas B, GonzÁLEZ G et al. Rotavirus-associated medical visits and hospitalizations in South America: a prospective study at three large sentinel hospitals. Pediatr Infect Dis J 2001; 20: 685-93.

6. WHO, Initiative for Vaccine Research. State of the art of the new vaccines research and development. 2003 Disponible en: http://www.who.int/ vaccine research/documents/en/stateofart excler.pdf. (Consultado el 03 de enero de 2005).

7. Glass RI, Bresee JS, Parashar UD, Jiang B, Gentsch $\mathrm{J}$. The future of rotavirus vaccines: a major setback leads to new opportunities. Lancet 2004; 363: 1547-50.

8. De Vos B, Vesikari T, Lunhares A, Salinas B, Pérez Schael I, Ruiz Palacios G et al. A rotavirus vaccine for prophylaxis of infants against rotavirus gastroenteritis. Pediatr Infect Dis J 2004; 23: S179-S182.

9. MiLER MA, MCCANN L Policy analysis of the use of hepatitis b, haemophilus influenzae type b-, Streptococcus pneumoniae-conjugate and rotavirus vaccines in national immunization schedules. Health Econ 2000; 9: 19-35.

10. Instituto Nacional de Estadísticas. Información Censo 2002. Disponible en: http://www.ine.cl (Consultado el 24 de febrero de 2005). información que puede ser de utilidad para la toma de decisiones en cuanto a la conveniencia de introducir una vacuna contra rotavirus en el calendario de vacunaciones.

11. Instituciones de Salud Previsional (ISAPRE), 2001. Disponible en: www.isapre.cl (Consultado el 24 de febrero de 2005).

12. O’Ryan M, Matson DO, Estes MK, Pickering LK. Molecular epidemiology of rotavirus in children attending day care centers in Houston. J Infect Dis 1990; 162: 810-16.

13. Minsal 2002 Disponible en http://deis.minsal.cl/ ev/def2002/t02_NATA.htm. (Consultado el 03 de enero del 2005).

14. Asociación Médica Mundial. Principios éticos para las investigaciones médicas en seres humanos. Disponible en: http://www.uchile.cl/bioeti$\mathrm{ca} /$ doc/helsinkrev.htm. (Consultado el 03 de enero de 2005).

15. International Conference on Harmonization of Technical Requirements for Registration of Pharmaceuticals for Human Use. Good Clinical Practice: Consolidated Guideline. Disponible en: http:/ /www.ncehr-cnerh.org/english/gcp/ (Consultado el 03 de enero de 2005).

16. O'Ryan MJ, Ramsay M, Brown D, Gay NJ, FarringTON CP, WALL PG. Hospital admissions attributable to rotavirus infection in England. J Infect Dis 1996; 174 (Suppl 1): S12-8.

17. MATSON DO, ESTES MK. Impact of rotavirus infection at a large pediatric hospital. J Infect Dis 1990; 162: 598-605.

18. Matson DO, O’Ryan ML, Jiang X, Mitchell DK. Rotavirus, enteric adenoviruses, caliciviruses, astroviruses, and other viruses causing gastroenteritis. In Clinical Virology Manual, $3^{\text {rd }}$ Edition. S Spector, RL Hodinka, SA Young, Editors. Washington DC; ASM Press, 2000, pp. 270-294.

19. O’Ryan M, Mamani N, Avendaño LF, Cohen J, Peña A, Viliarroel J et al. Tipos Antigénicos de Rotavirus Circulantes en Santiago, Chile. Rev Méd Chile 1995; 123: 549-59.

20. O'Ryan M, Mamani N, Avendaño LF, Cohen J, Peña A, Vimarroel J et al. Molecular Epidemiology of Human Rotaviruses in Santiago, Chile. Pediatr Infect Dis J 1997; 16: 305-11. 